\title{
Point-to-point control of a 2R planar horizontal underactuated manipulator
}

\author{
Arun D. Mahindrakar, Shodhan Rao, R.N. Banavar * \\ Systems and Control Engineering, Indian Institute of Technology, Bombay, Mumbai 400076, India
}

Received 15 February 2005; received in revised form 21 September 2005; accepted 14 October 2005

Available online 20 December 2005

\begin{abstract}
This paper presents a control strategy for a two-link manipulator with revolute joints $(2 \mathrm{R})$ with an unactuated second joint, wherein the motion of the system is confined to a horizontal plane. The model takes into account the frictional forces present in the system. The control objective is to move the end-effector from a given position to a target point. The methodology involves two stages. In the first stage a finite-time controller is used to move the passive link to its desired position. In the second stage, the first link is moved to its desired position keeping the second link at rest, using friction as a "brake" and subject to the constraint that the cross-coupling torque acting on the second joint does not exceed the static friction. (c) 2005 Published by Elsevier Ltd.
\end{abstract}

Keywords: Underactuated; Nonholonomic; Controllability; Point-to-point control

\section{Introduction}

Control of underactuated manipulators without potential/elastic forces has generated attention in the recent past. These systems present control challenges due to the loss of linear controllability and full statefeedback linearising property. Furthermore, these systems do not satisfy Brocketts's necessary condition [1] and thus there does not exist any continuous state-feedback control law that can asymptotically stabilize such a system at a given equilibrium.

Control of two-link manipulators moving in a horizontal plane has been studied by De Luca et al. [2,3]. They have used nilpotent approximations [4] to control the $2 \mathrm{R}$ manipulator moving in a horizontal plane with a single actuator at the first joint. This system is locally accessible, but the sufficient conditions for STLC are not satisfied. As pointed out in [5], existing control strategies for steering the system from certain configurations involve spinning maneuvers, thereby indicating the lack of STLC property for this system. Kobayashi et al. [6] have analyzed controllability of a planar underactuated manipulators with one free joint.

\footnotetext{
* Corresponding author. Tel.: +91 222576 7888; fax: +91222576 4885.

E-mail address: banavar@sc.iitb.ac.in (R.N. Banavar).
} 
For system that neglect friction, there exists no straightforward way of synthesizing control laws for the point-to-point control of the above kind of manipulators. In [7], Reyhanoglu et al. have proposed a discontinuous nonlinear feedback controller for a special class of underactuated systems with only one unactuated degree-of-freedom. The controller renders the closed-loop equilibrium at the origin globally attractive.

Recently, Bullo and Lynch [8] have introduced a new notion of controllability called kinematic controllability for underactuated mechanical systems. This property makes it possible to decouple trajectories between zero-velocity states. The property is established by defining a set of vector fields on the configuration space that span the entire configuration space. These vector fields, called the decoupling vector fields, can be used by the path planner to find paths that can be time-scaled without violating the acceleration level constraints. This approach has been used to design collision-free trajectory planning for the 3R manipulator [9].

In [10], the authors have applied an optimal control strategy, wherein the cost function considered is the reciprocal of the coupling index, a measure of the dynamic coupling available between the active and the passive joints of the manipulator. The authors Arai and his co-workers [11,12] have presented a control methodology for underactuated mechanical system with holding brakes on the passive joints. They exploit the dynamic coupling between the joints to achieve the controlled motion of the manipulator.

In practice, joint friction cannot be neglected in underactuated robotic manipulators, especially at the passive joint. This is because while friction at the active joints can be directly compensated, the same is not true for the passive joint. The models considered in $[2,13]$ neglect joint friction which is not easy to achieve in practice as it involves high manufacturing cost. Motivated by this problem, we propose a control strategy for a $2 \mathrm{R}$ underactuated system with friction at the joints.

\section{1. $2 R$ model with friction}

The configuration space of a $2 \mathrm{R}$ manipulator is $Q=\mathbb{S}^{1} \times \mathbb{S}^{1}$ and is parametrized by the coordinates $q=\left(q_{1}, q_{2}\right)$. The coordinates $q_{i}, i=1,2$ are the joint angles as shown in Fig. 1. The Euler-Lagrange equations of motion are

$$
M(q) \ddot{q}+h(q, \dot{q})=\tau
$$

where $\dot{q}$ and $\ddot{q}$ are the generalized velocities and generalized accelerations respectively. $M(q) \in \mathbb{R}^{2 \times 2}$ is the inertia matrix which is symmetric and positive definite. The centripetal and Coriolis terms are collected in

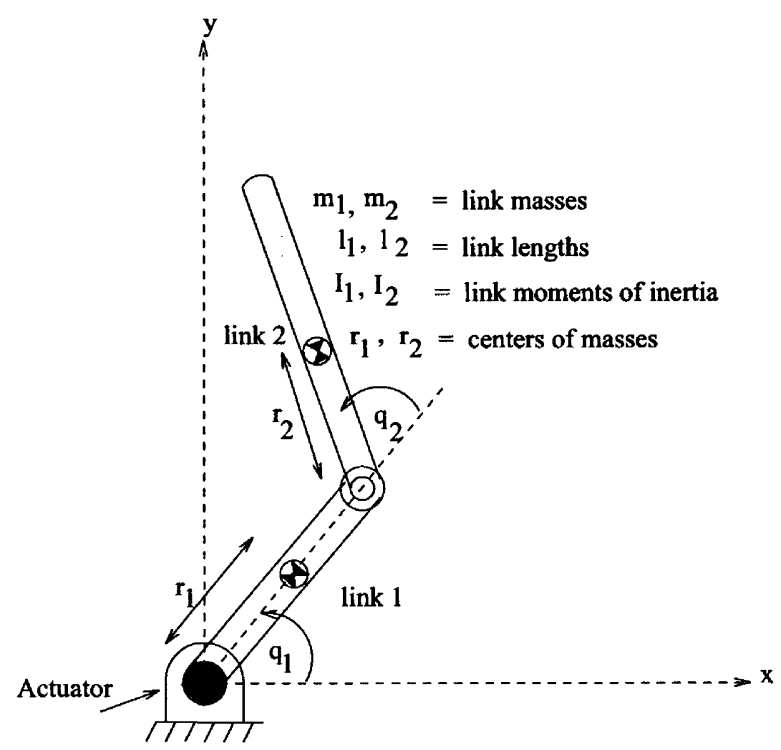

Fig. 1. Schematic of a $2 \mathrm{R}$ manipulator. 
the vector $h(q, \dot{q}) \in \mathbb{R}^{2}$. The vector $h$ contains terms purely quadratic in the velocities; gravity terms are absent since we assume that the manipulator moves in a horizontal plane. Define the following constants

$$
c_{1}=m_{1} r_{1}^{2}+m_{2} l_{1}^{2}+I_{1}, \quad c_{2}=m_{2} r_{2}^{2}+I_{2}, \quad c_{3}=m_{2} l_{1} r_{2} .
$$

The equations of motion accounting for the Coulomb plus viscous friction at the joints becomes

$$
\begin{aligned}
& m_{11} \ddot{q}_{1}+m_{12} \ddot{q}_{2}+h_{1}=\tau-\operatorname{SGN}\left(\dot{q}_{1}\right) F_{1}-b_{1} \dot{q}_{1}, \\
& m_{21} \ddot{q}_{1}+m_{22} \ddot{q}_{2}+h_{2}=-\operatorname{SGN}\left(\dot{q}_{2}\right) F_{2}-b_{2} \dot{q}_{2},
\end{aligned}
$$

where

$$
\begin{aligned}
& m_{11}=c_{1}+c_{2}+2 c_{3} \cos q_{2}, \quad m_{12}=c_{2}+c_{3} \cos q_{2}, \\
& m_{21}=m_{12}, \quad m_{22}=c_{2}, \\
& h_{1}=-c_{3}\left(2 \dot{q}_{1} \dot{q}_{2}+\dot{q}_{2}^{2}\right) \sin q_{2}, \quad h_{2}=c_{3} \dot{q}_{1}^{2} \sin q_{2}
\end{aligned}
$$

and $F_{i}, b_{i} \dot{q}_{i}, i=1,2$ represent the Coulomb and viscous friction forces respectively. The set-valued signum function is defined as

$$
\operatorname{SGN}(x) \begin{cases}\{1\} & \text { if } x>0 \\ \{-1\} & \text { if } x<0 \\ {[-1,1]} & \text { if } x=0\end{cases}
$$

We assume that the system has a strong inertial coupling $\left(m_{2} r_{2}^{2}+I_{2}>m_{2} l_{1} r_{2}\right)$. The equilibrium solutions of (4) with the external inputs equal to zero constitute an important class of solutions. If $(q, \dot{q})=\left(q^{\mathrm{e}}, 0\right)$ is an equilibrium solution, $q^{\mathrm{e}}$ is referred to as an equilibrium configuration. For the manipulator dynamics, the equilibrium configuration is given by $\left\{q \in Q: M(q)^{-1} h(q, 0)=0\right\}$. It is clear that all points $q \in Q$ are equilibrium configurations. The control objective is to move the links from rest to a target-configuration with zero-velocity.

\section{Controller design}

The control task involves the following two stages:

(i) Regulate $q_{2}$ to $q_{2 d}$, where $q_{2 d}$ is the desired position of the passive joint.

(ii) Regulate $q_{1}$ to $q_{1 d}$, where $q_{1 d}$ is the desired position of the active joint without affecting the position of the passive joint.

\subsection{Stage 1}

In this stage we further split-up the control task as follows:

(a) Move the second link to the desired position $q_{2 d}$ using the partially linearised model about the passive joint

$$
\begin{aligned}
& \ddot{q}_{1}=-m_{12}^{-1} m_{22} v_{2}-m_{12}^{-1}\left(h_{2}+\operatorname{SGN}\left(\dot{q}_{2}\right) F_{2}+b_{2} \dot{q}_{2}\right), \\
& \ddot{q}_{2}=v_{2},
\end{aligned}
$$

where the torque $\tau$ is related to the new input $v_{2}$ by

$$
\begin{aligned}
\tau= & \left(m_{12}-m_{11} m_{12}^{-1} m_{22}\right) v_{2}+\left(h_{1}+\operatorname{SGN}\left(\dot{q}_{1}\right) F_{1}+b_{1} \dot{q}_{1}\right) \\
& -m_{11} m_{12}^{-1}\left(h_{2}+\operatorname{SGN}\left(\dot{q}_{2}\right) F_{2}+b_{2} \dot{q}_{2}\right) .
\end{aligned}
$$

We steer $q_{2}$ to its desired position $q_{2 d}$ using a finite-time controller. Finite-time controllers are differential equations with the property that the origin is asymptotically stable, and all solutions which converge 
to zero do so in finite-time. We use the following continuous finite-time controller proposed by Bhat and Bernstein in [14]

$v_{2}=-\operatorname{sign}\left(\dot{q}_{2}\right)\left|\dot{q}_{2}\right|^{1 / 3}-\operatorname{sign}\left(S\left(q_{2}, \dot{q}_{2}\right)\right)\left|S\left(q_{2}, \dot{q}_{2}\right)\right|^{1 / 3}$,

where

$S\left(q_{2}, \dot{q}_{2}\right)=\left(\left(q_{2}-q_{2}^{\mathrm{d}}\right)+\frac{3}{5} \dot{q}_{2}^{5 / 3}\right)$

and the function sign $(\cdot)$ is defined as

$\operatorname{sign}(x)= \begin{cases}1 & \text { if } x>0, \\ -1 & \text { if } x<0, \\ 0 & \text { if } x=0,\end{cases}$

to achieve the desired objective. As $q_{2} \rightarrow q_{2 d}, \dot{q}_{2} \rightarrow 0$, the limiting behaviour of the system is given by $\left|m_{12} \ddot{q}_{1}+\left(c_{3} \sin q_{2 d}\right) \dot{q}_{1}^{2}\right|=F_{2}$.

(b) Once $\left(q_{2}=q_{2 d}, \dot{q}_{2}=0\right)$, the control task is to bring the first link to rest without disturbing the second link position. The following proposition brings out the condition under which this can be achieved.

Proposition 2.1. If the initial condition of the system is $\left(q_{2}=q_{2 d}, \dot{q}_{2}=0, \ddot{q}_{2}=0\right)$, then the second link continues to be at rest relative to the first link if and only if

$$
\left|m_{12} \ddot{q}_{1}+\left(c_{3} \sin q_{2 d}\right) \dot{q}_{1}^{2}\right| \leqslant F_{2} .
$$

Proof. With the second link at rest, the governing equation (3) reduces to

$$
m_{12} \ddot{q}_{1}+\left(c_{3} \sin q_{2 d}\right) \dot{q}_{1}^{2}=f_{2},
$$

where $f_{2}$ is the static friction acting at the second joint and $\left|f_{2}\right| \leqslant F_{2}$. The second link will continue to remain at rest if the absolute value of the static friction acting at the second joint is less than or equal to $F_{2}$ or $\left|f_{2}\right| \leqslant F_{2}$ from which (8) follows.

The first link is brought to rest without disturbing the second link using the following control law, which obeys (8).

$$
\tau= \begin{cases}\frac{-m_{11}}{m_{12}}\left(F_{2}-c+h_{2}\right)+F_{1}+b_{1} \dot{q}_{1}, & \dot{q}_{1}>\epsilon, \\ \frac{m_{11}}{m_{12}}\left(F_{2}-c-h_{2}\right)-F_{1}+b_{1} \dot{q}_{1}, & \dot{q}_{1}<\epsilon, \\ 0, & \dot{q}_{1} \in[-\epsilon, \epsilon],\end{cases}
$$

where $0<c<F_{2}$ and $\epsilon>0$ is small and is chosen such that chattering phenomenon is avoided. At the end of the first stage, the system is at rest with $q_{1}=q_{10}, q_{2}=q_{2 d}$.

\subsection{Stage 2}

The final task is to move the first link to the desired position $q_{1 d}$ without disturbing the second link position. The strategy involves accelerating and decelerating (if $q_{1 d}>q_{10}$, decelerating and accelerating otherwise) the first link such that the maximum magnitude $\left(\omega_{\max }\right)$ of the first link velocity and the magnitude $(\beta)$ of acceleration/deceleration satisfy the following constraints:

$$
\begin{aligned}
& \left|m_{12} \beta+\left(c_{3} \sin q_{2 d}\right) \omega_{\max }^{2}\right| \leqslant F_{2}-c, \\
& \left|m_{12} \beta-\left(c_{3} \sin q_{2 d}\right) \omega_{\text {max }}^{2}\right| \leqslant F_{2}-c .
\end{aligned}
$$

From Fig. 2, we have

$$
\omega_{\max }^{2}=\alpha s,
$$




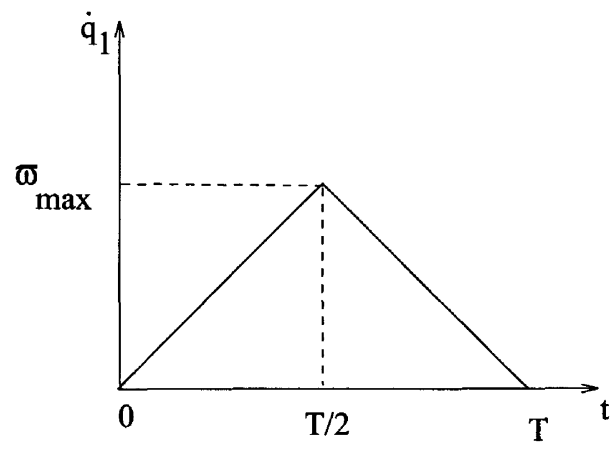

Fig. 2. Velocity profile.

where $\alpha$ is the angular acceleration of the first link and $s=q_{1 d}-q_{10}$. The variables $s$ and $\alpha$ are related to $T$ by $T=2 \sqrt{\frac{s}{\alpha}}$. We now have four possible cases and we propose a control law for each of the case as follows:

Case 1. $q_{1 d}>q_{10}$ and $\sin q_{2 d}>0$. Choose $\alpha$ such that the following is satisfied

$$
m_{12} \alpha+\left(c_{3} \sin q_{2 d}\right) \omega_{\max }^{2}=\left(F_{2}-c\right) .
$$

Eq. (14) ensures that the constraint (12) is not violated for $t \in[T / 2, T]$. From (13) and (14), we have

$$
\alpha=\frac{\left(F_{2}-c\right)}{m_{12}+s\left(c_{3} \sin q_{2 d}\right)} .
$$

The control law takes the form

$$
\tau= \begin{cases}m_{11} \alpha+F_{1}+b_{1} \alpha t, & t \in\left[0, \frac{T}{2}\right], \\ -m_{11} \alpha+F_{1}+b_{1} \alpha(T-t), & t \in\left[\frac{T}{2}, T\right], \\ 0, & t>T .\end{cases}
$$

Case 2. $q_{1 d}<q_{10}$ and $\sin q_{2 d}>0$.

Choose $\alpha$ such that the following is satisfied

$$
-m_{12} \alpha+\left(c_{3} \sin q_{2 d}\right) \omega_{\max }^{2}=\left(F_{2}-c\right) .
$$

Eq. (17) ensures that the constraint (11) is not violated for $t \in[T / 2, T]$. From (13) and (17), we have

$$
\alpha=\frac{-\left(F_{2}-c\right)}{m_{12}-s\left(c_{3} \sin q_{2 d}\right)}
$$

Here, the control law has the form

$$
\tau= \begin{cases}m_{11} \alpha-F_{1}+b_{1} \alpha t, & t \in\left[0, \frac{T}{2}\right], \\ -m_{11} \alpha-F_{1}+b_{1} \alpha(T-t), & t \in\left[\frac{T}{2}, T\right], \\ 0, & t>T .\end{cases}
$$

Case 3. $q_{1 d}>q_{10}$ and $\sin q_{2 d}<0$.

Choose $\alpha$ such that the following is satisfied

$$
-m_{12} \alpha+\left(c_{3} \sin q_{2 d}\right) \omega_{\max }^{2}=-\left(F_{2}-c\right) .
$$

Eq. (20) ensures that the constraint (11) is not violated. From (13) and (20), we have

$$
\alpha=\frac{\left(F_{2}-c\right)}{m_{12}-s\left(c_{3} \sin q_{2 d}\right)}
$$


and the control law has the form

$$
\tau= \begin{cases}m_{11} \alpha+F_{1}+b_{1} \alpha t, & t \in\left[0, \frac{T}{2}\right], \\ -m_{11} \alpha+F_{1}+b_{1} \alpha(T-t), & t \in\left[\frac{T}{2}, T\right], \\ 0, & t>T .\end{cases}
$$

Case 4. $q_{1 d}<q_{10}$ and $\sin q_{2 d}<0$.

Choose $a$ such that the following is satisfied

$$
m_{12} \alpha+\left(c_{3} \sin q_{2 d}\right) \omega_{\max }^{2}=-\left(F_{2}-c\right) .
$$

Eq. (23) ensures that the constraint (12) is not violated. From (13) and (23), we have

$$
\alpha=\frac{-\left(F_{2}-c\right)}{m_{12}+s\left(c_{3} \sin q_{2 d}\right)} .
$$

We note that the constraint (12) is not violated for $t \in[T / 2, T]$. Finally, the control law has the form

$$
\tau= \begin{cases}m_{11} \alpha-F_{1}+b_{1} \alpha t, & t \in\left[0, \frac{T}{2}\right], \\ -m_{11} \alpha-F_{1}+b_{1} \alpha(T-t), & t \in\left[\frac{T}{2}, T\right], \\ 0, & t>T .\end{cases}
$$
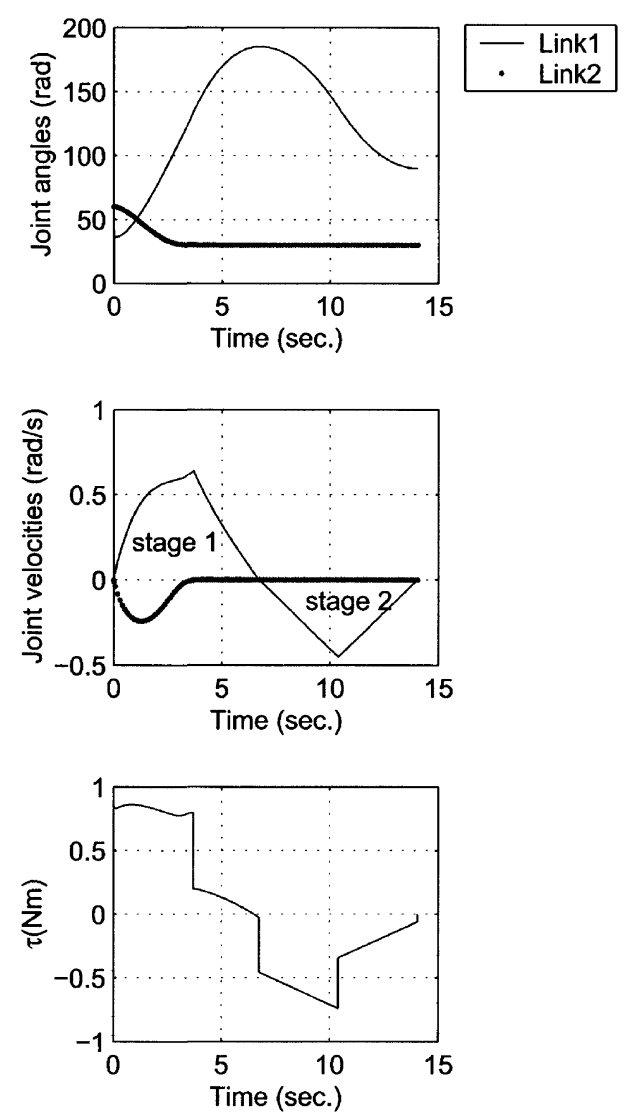

Fig. 3. Time response for the case: $q_{1 d}<q_{10}$ and $\sin q_{2 d}>0$. 


\section{Simulations}

The simulations were performed on SIMULINK. The manipulator parameters used are $c_{1}=0.725$, $c_{2}=0.3179, c_{3}=0.3147, F_{1}=0.26, F_{2}=0.116, b_{1}=0.6236, b_{2}=0.1223$. The initial values are chosen as $\left(q_{1}(0)=\pi / 5, q_{2}(0)=\pi / 4, \dot{q}_{1}(0)=0, \dot{q}_{2}(0)=0\right)$ while the desired values are $\left(q_{1 d}=\pi, q_{2 d}=\pi / 6, \dot{q}_{1 d}=\right.$ $\left.0, q_{2 d}=0\right)$. The values of $c$ and $\epsilon$ are fixed at 0.01 . Fig. 3 shows the simulation results for the first and the second stages.

\section{Conclusions}

We have presented a control strategy to control an underactuated $2 \mathrm{R}$ manipulator with friction at both the joints. The strategy consists of two stages. In the first stage the second joint angle is brought to its desired position by imparting appropriate motion to the first link. In the second stage, static friction at the second joint is made use of in bringing the first link to its desired position without disturbing the second link. Simulation results demonstrate the effectiveness of the control strategy.

\section{Acknowledgement}

This work was sponsored by the Science and Engineering research Council, Department of Science and Technology, India under a research project (100/IFD/2008/2001-02).

\section{References}

[1] R.W. Brockett, Asymptotic stability and feedback stabilization, in: R.W. Brockett, R.S. Millman, H.J. Sussmann (Eds.), Differential Geometric Control Theory, Birkhauser, Boston, MA, 1983, pp. 181-191.

[2] A.D. Luca, R. Mattone, G. Oriolo, Stabilization of an underactuated planar 2R manipulator, International Journal of Robust and Nonlinear Control 24 (July) (2000) 181-198.

[3] A.D. Luca, G. Oriolo, Trajectory planning and control for planar robots with passive last joint, International Journal of Robotics Research 21 (5-6) (2002) 575-590.

[4] H. Hermes, Nilpotent and high-order approximations of vector field systems, SIAM Review 33 (2) (1991) $238-264$.

[5] A.D. Luca, S. Iannitti, A simple STLC test for mechanical systems under-actuated by one control, in: Proceedings of the IEEE International Conference on Robotics and Automation, Washington, DC, May 2002, pp. 1735-1740.

[6] K. Kobayashi, T. Yoshikawa, Controllability of underactuated manipulators with one unactuated joint, International Journal of Robotics Research 21 (5-6) (2002) 555-561.

[7] M. Reyhanoglu, S. Cho, N.H. McClamroch, Discontinuous feedback control of a special class of underactuated mechanical systems, International Journal of Robust and Nonlinear Control 24 (July) (2000) 265-281.

[8] F. Bullo, K.M. Lynch, Kinematic controllability for decoupled trajectory planning in underactuated mechanical systems, IEEE Transactions on Robotics \& Automation 17 (August) (2001) 402-412.

[9] K.M. Lynch, N. Shiroma, H. Arai, K. Tanie, Collision-free trajectory planning for a 3-DOF robot with a passive joint, International Journal of Robot Research 19 (12) (2000) 1171-1184.

[10] M. Bergerman, Y. Xu, Optimal control sequence for underactuated manipulators, in: Proceedings of the IEEE International Conference on Robotics and Automation, Minneapolis, USA, April 1996, pp. 3714-3719.

[11] H. Arai, S. Tachi, Position control of a manipulator with passive joints using dynamic coupling, IEEE Transactions on Robotics \& Automation 7 (August) (1991) 528-534.

[12] H. Arai, K. Tanie, S. Tachi, Dynamic control of a manipulator with passive joints in operational space, IEEE Transactions on Robotics \& Automation 9 (February) (1993) 85-93.

[13] H. Arai, K. Tanie, N. Shiroma, Nonholonomic control of a three-dof planar underactuated manipulator, IEEE Transactions on Robotics \& Automation 14 (October) (1998) 681-695.

[14] S.P. Bhat, D.S. Bernstein, Continuous finite-time stabilization of the translational and rotational double integrators, IEEE Transactions on Automatic Control 43 (May) (1998) 678-682. 